\title{
On the implicit bond-dependency origins of bridge interactions
}

\author{
Roman F. Nalewajski • Piotr Gurdek
}

Received: 12 December 2010 / Accepted: 24 March 2011 / Published online: 11 April 2011

(C) The Author(s) 2011. This article is published with open access at Springerlink.com

\begin{abstract}
The indirect (through-bridge) components of chemical interactions between atomic orbitals (AO) are shown to originate from the indirect dependencies between $\mathrm{AO}$ due to the orbital intermediaries in the bond system of the molecule. They are expressed in terms of the bridge-coupling elements of the density matrix via the chain rule transformation of the implicit derivatives between the indirectly bonded $\mathrm{AO}$ in the molecular bond system. The elements of the charge-and-bond-order (CBO) matrix are interpreted as the canonical derivatives between the AO-projections onto the bond subspace combining the occupied Molecular Orbitals (MO). The chain-rule manipulations are then used to express the scattering amplitudes via AO intermediaries in terms of the relevant elements of the CBO matrix. The squares of such amplitudes are related to the Wiberg-type indirect bond components, which complement the familiar direct Wiberg bond-order contributions. The interference implications of the probability scatterings via the multiple cascades involving all basis functions are examined. These probability propagations are shown to preserve the stationary conditional probabilities of the underlying molecular communication channel in AO resolution.
\end{abstract}

Keywords Bond orders · Chemical interactions · Direct/indirect bonds · Information-theoretic bond multiplicities · Interference of molecular communications · Orbital Communication Theory · Wiberg bond order

Throughout the paper $A$ denotes a scalar quantity, $\boldsymbol{A}$ stands for a row-vector, and $\mathbf{A}$ represents a square or rectangular matrix.

R. F. Nalewajski $(\bowtie) \cdot$ P. Gurdek

Theoretical Chemistry Department, Jagiellonian University, R. Ingardena 3, 30-060 Cracow, Poland e-mail: nalewajs@chemia.uj.edu.pl 


\section{Introduction}

The key concepts and techniques of Information Theory (IT) [1-4] used to characterize the ordinary communication systems have been successfully applied to explore the bonding structure of molecules $[5,6]$ in terms of both the overall bond multiplicity, its ionic/covalent components, and through the corresponding entropy/information descriptors of the localized (diatomic) chemical interactions [6-8]. This Communication Theory of the Chemical Bond (CTCB) introduces the key concept of the molecular information channel [9] in which the electron probabilities are propagated between Atoms-in-Molecules (AIM) [5] or Atomic Orbitals (AO) [6]. It has been shown to provide a novel perspective on the entropic origins of the bond multiplicity (order) $[5,6]$. The communication-noise (covalency) and information-flow (ionic) descriptors (in bits) of the orbitally-resolved channels of the Orbital Communication Theory (OCT) $[6,10-12]$ and the associated overall IT bond-order were shown to reflect the chemical intuition quite well. The localized bond-multiplicities between the given pair of Atoms-in-Molecules (AIM) [6,7] were shown to reproduce the quadratic Wiberg index [13] of quantum chemistry in diatomic systems and to closely approximate the latter in typical polyatomic molecules, at the same time providing it the covalent/ionic resolution [6-8]. The many-orbital generalization of OCT $[14,15]$, which allows one to account for the inter-bond coupling phenomena, opens the IT-perspective on the origins of catalytical activity, multi-bond reactivity, poly-center bonds, etc. [6].

The chemical interaction between the specified pair of bonded atoms has recently been shown to exhibit both the through-space and, hitherto neglected, through-bridge components [16-18]. The former reflects the direct interactions between AO while the latter is realized indirectly, through the remaining basis functions which constitute an effective bridge for an implicit chemical coupling between orbitals contributed by more distant atoms. The most efficient bridges for such an implicit bonding mechanism are the real chemical bridges, originating from the basis functions contributed by the chemically bonded atoms connecting the given "terminal" atoms in question.

Each pair of AO thus exhibits partial through-space and through-bridge bond components. The "order" of the former quickly vanishes with increasing inter-atomic separation. It is also small when the interacting AO are heavily engaged in forming chemical bonds with other atoms or remain non-bonding thus describing the lone electron pairs. In these cases the chemical interaction can still assume appreciable values when the remaining atoms form an effective bridge of the neighboring, chemically bonded atoms, which links the two AO in question.

Thus, a non-vanishing density-matrix element coupling the two AO in the molecule, which in MO theory reflects their directly-bonding status, is not essential for the existence of their through-bridge interaction. The latter may exist even when the direct interaction vanishes provided the two AO strongly couple to the chemically bonded chain of orbital intermediaries connecting them. This novel, indirect ("throughbridge") mechanism of bonding interactions in molecules, first conjectured to explain the bonding patterns of small propellane systems [5], generates the bond-order contributions complementary to those resulting from the familiar direct ("through-space") interactions between the basis functions used to represent the occupied Molecular Orbitals (MO), which determine the system chemical bonds. 
In particular, it has been demonstrated using both the generalized Wiberg bond orders and the associated OCT bond multiplicities how atoms exhibiting the vanishing direct chemical interaction can be still bonded indirectly, via the AO/AIM bridges $[16,17]$. For example, this novel mechanism has been shown to have important implications for the bonding patterns of $\pi$-interactions in hydrocarbons. In the $\pi$-system of benzene the neighboring ortho-carbons exhibit a strong Wiberg bondmultiplicity almost exclusively of the through-space origin, while the cross-ring interactions, between the meta- and para-carbons, where shown to be described by much smaller but practically equalized resultant bond-orders, being distinguished solely by the direct/indirect composition of these overall chemical interactions. More specifically, the meta bonds have been shown to be realized exclusively through bridges, while the para bonds exhibit a comparable direct and indirect components.

In OCT the direct bond between the specified pair of interacting AO which constructively mix into the bonding MO originates from their mutual probability scattering in the molecule. Its covalency represents a finite conditional probability due to these molecular communications, related to the square of the corresponding element of the system Charge-and-Bond-Order (CBO) matrix coupling the two basis functions, and hence also - to the associated Wiberg bond-order contribution. These direct AO communications are in accordance with the electron delocalization pattern implied by the system occupied (bonding) subspace of MO. The "implicit" (through-bridge) bond component can be similarly viewed as resulting from the indirect information propagation via the bridging AO. Therefore, while the through-space bonding reflects in OCT a direct "conversation" between AO, the through-bridge bonding can be compared to a "hearsay" spreading between between two AO in question via the connecting chain of orbital intermediaries involved in the effective communication chain under consideration.

One thus distinguishes in OCT the direct ("dialogue") and indirect ("gossip") contributions to the effective IT bond order, which together determine the resultant bond multiplicity between the given pair of AO or AIM. The direct (explicit) bonding interaction between neighboring atoms, reflected by the original Wiberg bond-orders, is generally associated with the presence of the bond-charge or the increase of information density between the two nuclei. However, for more distant atomic partners such an accumulation of valence electrons can be absent, e.g., in the cross-ring $\pi$-interactions in benzene or between the bridgehead carbon atoms in small propellanes, for which the "charge-shift" bonding mechanism [19] has been proposed within the familiar Valence-Bond (VB) description of molecular systems. The latter involves instantaneous charge fluctuations due to a strong resonance between covalent and ionic VB structures. As we have argued elsewhere [16], such an indirect (implicit) bonding interaction lacking an accumulation of the bond-charge (information) can be also realized indirectly, through the neighboring AO intermediaries forming a bridge for an effective interaction (communication) between more distant AO.

The through-bridge mechanism was conjectured to result from the implicit dependencies between the (non-orthogonal) AO projections into the bonding subspace of the occupied MO [16-18]. These AO components reflect the joint participation of the basis functions in the whole system of chemical bonds. It is the main purpose of the present work to explore the $\mathrm{CBO}$ framework of these indirect dependencies in a 
more detail and to demonstrate that the novel through-bridge mechanism represents a natural extension of the direct dependencies already manifested in the through-space bond components.

\section{Density matrix elements as the bond-projected derivatives of orbitals}

In the orbital approximation of the spin-Restricted Hartree-Fock (RHF) description of the standard SCF MO theory of the (closed-shell) ground-state configuration of the molecular system containing $N=2 n$ electrons the lowest $n$ (doubly occupied) orthonormal MO $\left|\varphi^{\circ}\right\rangle$ determine the system network of chemical bonds. In the LCAO MO approach the whole set of MO, $|\varphi\rangle=\left(\left|\varphi^{o}\right\rangle,\left|\varphi^{v}\right\rangle\right)$, where $\left|\varphi^{v}\right\rangle$ groups the remaining (virtual) MO, is generated as linear combinations of the (Löwdin-orthogonalized) AO, $|\chi\rangle=\left\{\left|\chi_{i}\right\rangle \equiv|i\rangle\right\}=(|1\rangle,|2\rangle, \ldots,|m\rangle),\langle\chi \mid \chi\rangle=\left\{\delta_{i, j}\right\} \equiv \mathbf{I}$, contributed by the system constituent atoms:

$$
\begin{aligned}
|\boldsymbol{\varphi}\rangle & =\left\{\left|\varphi_{s}\right\rangle \equiv|s\rangle\right\}=\left[\left(\left|\varphi_{1}\right\rangle,\left|\varphi_{2}\right\rangle, \ldots,\left|\varphi_{n}\right\rangle\right),\left(\left|\varphi_{n+1}\right\rangle, \ldots,\left|\varphi_{m}\right\rangle\right)\right] \\
& \equiv\left(\left|\varphi^{o}\right\rangle,\left|\varphi^{v}\right\rangle\right)=|\chi\rangle \mathbf{C}=|\chi\rangle\left(\mathbf{C}^{o} \mid \mathbf{C}^{v}\right) .
\end{aligned}
$$

Here, the rectangular matrices $\mathbf{C}^{o}=\left\langle\chi \mid \varphi^{o}\right\rangle$ and $\mathbf{C}^{v}=\left\langle\chi \mid \varphi^{v}\right\rangle$ group the expansion (LCAO) coefficients of the $n$ occupied and $(m-n)$ virtual $\mathrm{MO}$, respectively, to be determined from the iterative self-consistent-field (SCF) procedure. The full SCF LCAO MO matrix $\mathbf{C}$ is unitary, $\mathbf{C}^{\dagger}=\mathbf{C}^{-1}$, since it "rotates" orthonormal AO into the orthonormal MO, and hence the inverse transformation reads: $|\chi\rangle=|\boldsymbol{\varphi}\rangle \mathbf{C}^{\dagger}$.

The basis set projections onto the bond subspace $\left|\varphi^{o}\right\rangle$,

$$
\left|\chi^{b}\right\rangle=\left|\varphi^{o}\right\rangle\left\langle\varphi^{o} \mid \chi\right\rangle \equiv \hat{\mathrm{P}}_{\varphi}^{o}|\chi\rangle=\left|\varphi^{o}\right\rangle \mathbf{C}^{o \dagger}=\left\{\hat{\mathrm{P}}_{\varphi}^{o}\left|\chi_{i}\right\rangle=\left|i^{b}\right\rangle\right\}
$$

subsequently determine the CBO matrix $\gamma=2\left\langle\chi\left|\hat{\mathrm{P}}_{\varphi}^{o}\right| \chi\right\rangle=2 \mathbf{d}$ and the associated density matrix $\mathbf{d}=\left\langle\chi\left|\hat{\mathrm{P}}_{\varphi}^{o}\right| \chi\right\rangle=\left\langle\chi \mid \varphi^{o}\right\rangle\left\langle\varphi^{o} \mid \chi\right\rangle=\mathbf{C}^{o} \mathbf{C}^{o \dagger}$. The latter is seen to represent the overlap matrix between the AO projections into the bonding subspace of MO:

$$
\mathbf{d}=\left\langle\chi\left|\hat{\mathrm{P}}_{\varphi}^{o}\right| \chi\right\rangle=\left(\langle\chi| \hat{\mathrm{P}}_{\varphi}^{o}\right)\left(\hat{\mathrm{P}}_{\varphi}^{o}|\chi\rangle\right)=\left\langle\chi^{b} \mid \chi^{b}\right\rangle
$$

where we have used the idempotency relation $\left(\hat{\mathrm{P}}_{\varphi}^{o}\right)^{2}=\hat{\mathrm{P}}_{\varphi}^{o}$, which is also reflected by the associated idempotency relations for $\gamma$ and $\mathbf{d}$ :

$$
\gamma^{2}=4\left\langle\chi\left|\hat{\mathrm{P}}_{\varphi}^{o}\right| \chi\right\rangle\left\langle\chi\left|\hat{\mathrm{P}}_{\varphi}^{o}\right| \chi\right\rangle=4\left\langle\chi\left|\left(\hat{\mathrm{P}}_{\varphi}^{o}\right)^{2}\right| \chi\right\rangle=4\left\langle\chi\left|\hat{\mathrm{P}}_{\varphi}^{o}\right| \chi\right\rangle=2 \gamma \quad \text { or } \quad \mathbf{d}^{2}=\mathbf{d}
$$

The CBO matrix reflects the promoted, valence state of AO in the molecule, with the diagonal elements measuring the effective electron occupations of these basis functions, $\left\{N_{i}=\gamma_{i, i}=2\left\langle i^{b} \mid i^{b}\right\rangle\right\}$, tr $\boldsymbol{\gamma}=N$. The off-diagonal CBO elements between AO 
on different atoms similarly reflect the bonding status of the direct chemical interaction of the specified AO pair in the molecule, with the positive (negative) values signifying the resultant bonding (anti-bonding) coupling between the two basis functions in question, and the vanishing bond-order identifying their directly non-bonding status. Thus, for the positive AO overlap the resultant "constructive" (bonding) interference between two AO implies the positive (in-phase) product of their direct bond-projections, while its negative (out-of-phase) value identifies their resultant "destructive" interference in the molecule.

It also follows from Eq. 2 that the bond-projections $\left|\chi^{b}\right\rangle$ of basis functions can be expressed in terms of the $\mathrm{AO}$ themselves:

$$
\left|\chi^{b}\right\rangle=\left|\varphi^{o}\right\rangle \mathbf{C}^{o \dagger}=|\chi\rangle\left(\mathbf{C}^{o} \mathbf{C}^{o \dagger}\right)=|\chi\rangle \mathbf{d},
$$

and hence the bond-overlap (density) matrix $\mathbf{d}$ can be interpreted as matrix combining the derivatives

$$
\mathbf{d}=\frac{\partial \chi^{b}}{\partial \chi}=\left\{d_{i, j}=\left\langle i^{b} \mid j^{b}\right\rangle=\left(\frac{\partial \chi_{j}^{b}}{\partial \chi_{i}}\right)\right\} .
$$

Moreover, using the idempotency relation of Eq. 4 allows one to interpret Eq. 5 as the linear transformation of the AO projections themselves:

$$
\left|\chi^{b}\right\rangle=(|\chi\rangle \mathbf{d}) \mathbf{d}=\left|\chi^{b}\right\rangle \mathbf{d}
$$

Therefore the partial derivatives of Eq. 6 can be also interpreted as reflecting the linear dependencies between the bond projections of the basis functions:

$$
\mathbf{d}=\frac{\partial \chi^{b}}{\partial \chi^{b}}=\left\{d_{i, j}=\left(\frac{\partial \chi_{j}^{b}}{\partial \chi_{i}^{b}}\right)\right\} .
$$

This allows one to interpret the idempotency relation as the chain-rule identity:

$$
\left(\mathbf{d}^{2}\right)_{i, j}=\sum_{k}\left(\frac{\partial \chi_{k}^{b}}{\partial \chi_{i}^{b}}\right)\left(\frac{\partial \chi_{j}^{b}}{\partial \chi_{k}^{b}}\right)=\left(\frac{\partial \chi_{j}^{b}}{\partial \chi_{i}^{b}}\right)=(\mathbf{d})_{i, j}
$$

In fact, using the resolution of the identity operator into complementary projections onto the mutually orthogonal subspaces of the occupied and virtual MO,

$$
1=\left|\varphi^{o}\right\rangle\left\langle\varphi^{o}|+| \varphi^{v}\right\rangle\left\langle\varphi^{v}\right| \equiv \hat{\mathrm{P}}_{\varphi}^{o}+\hat{\mathrm{P}}_{\varphi}^{v}, \quad \hat{\mathrm{P}}_{\varphi}^{o} \hat{\mathrm{P}}_{\varphi}^{v}=0
$$

gives the associated resolution of the basis functions:

$$
|\chi\rangle=\hat{\mathrm{P}}_{\varphi}^{o}|\chi\rangle+\hat{\mathrm{P}}_{\varphi}^{v}|\chi\rangle=\left|\chi^{b}\right\rangle+\left|\chi^{v}\right\rangle
$$


This allows one to partition the unit matrix of AO-overlap into the complementary contributions originating from these two subspaces of MO:

$$
\begin{aligned}
\langle\chi \mid \chi\rangle & =\frac{\partial \chi}{\partial \chi}=\mathbf{I}=\left(\left\langle\chi^{b}\left|+\left\langle\chi^{v}\right|\right)\left(\left|\chi^{b}\right\rangle+\left|\chi^{v}\right\rangle\right)\right.\right. \\
& =\left\langle\chi^{b} \mid \chi^{b}\right\rangle+\left\langle\chi^{v} \mid \chi^{v}\right\rangle=\frac{\partial \chi^{b}}{\partial \chi^{b}}+\frac{\partial \chi^{v}}{\partial \chi^{v}} \\
& =\left(\left\langle\chi^{b}\left|+\left\langle\chi^{v}\right|\right) \mid \chi\right\rangle=\frac{\partial \chi}{\partial \chi^{b}}+\frac{\partial \chi}{\partial \chi^{v}}\right. \\
& =\left\langle\chi \left|\left(\left|\chi^{b}\right\rangle+\left|\chi^{v}\right\rangle\right)=\frac{\partial \chi^{b}}{\partial \chi}+\frac{\partial \chi^{v}}{\partial \chi} .\right.\right.
\end{aligned}
$$

These subspace-overlaps thus define the partial orbital dependencies in the two subsets of MO:

$$
\left(\frac{\partial \chi_{j}^{b}}{\partial \chi_{i}^{b}}\right)=\left(\frac{\partial \chi_{j}^{b}}{\partial \chi_{i}}\right)=\left(\frac{\partial \chi_{j}}{\partial \chi_{i}^{b}}\right) \text { and }\left(\frac{\partial \chi_{j}^{v}}{\partial \chi_{i}^{v}}\right)=\left(\frac{\partial \chi_{j}^{v}}{\partial \chi_{i}}\right)=\left(\frac{\partial \chi_{j}}{\partial \chi_{i}^{v}}\right)
$$

We finally recall that the density matrix also determines the conditional probabilities for the direct information propagation in the AO information system [5,10-12], the key concept of OCT, in which the basis functions of SCF MO calculations provide a natural resolution level of the electron-assignment "events", appropriate for discussing the information scattering via the system chemical bonds. This AO communication network is then described by standard quantities developed in IT for real communication devices [1-4]. Due to electron delocalization throughout the network of chemical bonds the transmission of "signals" about the electron-assignments to AO becomes randomly disturbed in the molecule, thus exhibiting typical communication "noise". Indeed, an electron initially attributed to the given AO in the channel "input" $\mathbf{a}=\left\{\chi_{i}\right\}$ can be later found with a non-zero probability at several locations in the molecular "output" $\mathbf{b}=\left\{\chi_{j}\right\}$. This feature of the electron delocalization is embodied in the (direct) conditional probabilities of the "outputs-given-inputs",

$$
\begin{aligned}
\mathbf{P}(\mathbf{b} \mid \mathbf{a}) & =\left\{P(j \mid i)=\left(2 \gamma_{i, i}\right)^{-1} \gamma_{i, j} \gamma_{j, i}=\left(2 \gamma_{i, i}\right)^{-1}\left|\gamma_{i, j}\right|^{2}=\left(d_{i, i}\right)^{-1}\left|d_{i, j}\right|^{2}\right. \\
& \left.\equiv A(j \mid i) A(j \mid i)^{*} \equiv\left|A_{i \rightarrow j}\right|^{2} \equiv P(i \rightarrow j)\right\},
\end{aligned}
$$

where the normalization constant results from the requirement $\sum_{j} P(j \mid i)=1$. They have been determined [10] from the superposition principle of quantum mechanics [20] supplemented by the "physical" projection onto the bond subspace of the occupied MO. The preceding equation also introduces the quantum-mechanical amplitude $A(j \mid i) \equiv A_{i \rightarrow j}$ associated with the conditional probability $P(j \mid i) \equiv P_{i \rightarrow j}$. It is seen to be determined by the corresponding (occupation renormalized) element of the $\mathrm{CBO} /$ density matrix:

$$
A_{i \rightarrow j}=\gamma_{i, j} / \sqrt{2 \gamma_{i, i}}=d_{i, j} / \sqrt{d_{i, i}} \equiv N_{i \rightarrow j} d_{i, j} .
$$


It also follows from Eq. (14) that this direct conditional probability is related to the Wiberg bond-order $M_{i, j}=\left(\gamma_{i, j}\right)^{2}$,

$$
P(i \rightarrow j)=M_{i, j}\left(N_{i \rightarrow j}\right)^{2} / 4
$$

\section{Implicit-dependency origins of the bridge bonds}

The Wiberg bond-order measure between two basis functions $|i\rangle$ and $|j\rangle$ makes use of only their explicit dependency in the molecular bond system, reflected by the coupling CBO matrix element $\gamma_{i, j}=2\left\langle i^{b} \mid j^{b}\right\rangle=2 d_{i, j}$. It neglects all the remaining constraints, embodied by other $\mathrm{CBO}$ matrix elements involving these two $\mathrm{AO}$, which introduce the implicit dependencies between the two AO in question through the remaining orbitals participating in the bond subspace of MO. These indirect relations are responsible for the bridge contributions to the overall bond multiplicity between the specified pair of AO [16-18]. Indeed, the mutually-bonding status of two basis functions can be felt even at large distances, due to their coupling to the chain the chemically interacting AO intermediaries.

More specifically, since the bond-overlaps of Eq. 3 can be interpreted as derivatives between AO projections in the bond system of the molecule, $d_{i, j}=\gamma_{i, j} / 2=$ $\partial \chi_{j}^{b} / \partial \chi_{i}^{b}$, satisfying the associated chain (idempotency) rule of Eq. $9, \sum_{k} d_{i, k} d_{k, j}=$ $d_{i, j}$, one can express any displacement in one bond-projection in terms of displacements of all basis set projections:

$$
\delta \chi_{k}^{b}=\sum_{l} \delta \chi_{l}^{b} d_{l, k}
$$

In probing the bond-dependencies between the given pair $(i, j)$ of $\mathrm{AO}$ the Wiberg approach makes use of only the direct terms $d_{j, i}=d_{i, j}$ in the expansions of bond components of both basis functions involved, while neglecting the implicit dependencies reflected by the remaining derivatives. The latter are taken into account only in the indirect bond components, due to the bond projections of all remaining orbitals.

As indicated in Eqs. 14 and 16, the quadratic Wiberg indices $M_{i, j}$, of the direct chemical bond multiplicity, or the corresponding IT descriptors of the explicit bond order, and their through-bridge generalizations are related to the molecular probabilities measuring squares of the associated scattering amplitudes. The molecular (stationary) probability distribution in the multiple probability scatterings between the elementary AO states has been reconstructed by the interference of amplitudes of the generalized, multiple (cascade) scatterings through all the basis functions at arbitrary bridge-order [18]. Only the wave-like superposition of the sub-channel scattering amplitudes was shown to conserve the molecular (stationary) conditional probabilities of Eq. 14 at any bridge order. This property demonstrates that OCT provides the internally consistent quantum description of the molecular electronic structure and of the elementary AO communications, provided that the elementary scattering amplitudes are superimposed. The operator representation of such an information scattering process has facilitated the vector interpretation of the probability amplitudes 
as projections of the forward- and reverse-scattered states, eventually leading to the establishment of the independent modes (standing ways) of the molecular conditionalprobability propagation between basis functions, linked to the eigenvalue problem of the associated AO-communication operator [18].

The amplitudes of the through-bridge probability propagations can be explicitly expressed in terms of the implicit derivatives of Eqs. 6, 8 and 12, i.e., the density matrix elements, by using the chain rules of Eq. 9. Consider the simplest case of a single AO bridge in the information scattering between $|i\rangle$ and $|j\rangle$ through $|k\rangle, k \neq$ $(i, j), i \rightarrow k \rightarrow j$, reflected by the associated conditional bridge-probability

$$
\begin{aligned}
P(i \rightarrow j \mid k) & =\left|A_{(i \rightarrow j \mid k)}\right|^{2}=\left(d_{i, k} d_{k, j}\right)^{2} /\left(d_{i, i} d_{k, k}\right)=\left(d_{i, k} / \sqrt{d_{i, i}}\right)^{2}\left(d_{k, j} / \sqrt{d_{k, k}}\right)^{2} \\
& =\left|A_{i \rightarrow k}\right|^{2}\left|A_{k \rightarrow j}\right|^{2}=P(i \rightarrow k) P(k \rightarrow j) .
\end{aligned}
$$

It is defined by the associated amplitude, the renormalized implicit derivative of $\chi_{j}^{b}$ on $\chi_{i}^{b}$ through $\chi_{k}^{b}$,

$$
A_{(i \rightarrow j \mid k)}=N_{(i \rightarrow j \mid k)}\left(\frac{\partial \chi_{k}^{b}}{\partial \chi_{i}^{b}}\right)\left(\frac{\partial \chi_{j}^{b}}{\partial \chi_{k}^{b}}\right)=N_{(i \rightarrow j \mid k)} d_{i, k} d_{k, j},
$$

with the normalization constant,

$$
N_{(i \rightarrow j \mid k)}=1 /\left(d_{i, i} d_{k, k}\right)^{1 / 2},
$$

fixed to satisfy the relevant sum rule for conditional probabilities:

$$
\sum_{j} P(i \rightarrow j \mid k)=P(k \mid i) \equiv P(i \rightarrow k) .
$$

Indeed, the summation of the conditional probabilities over all possible final outputs $\{j\}$ in the sequential scatterings $i \rightarrow k \rightarrow\{j\}$ must reproduce the conditional probability of its first step $i \rightarrow k$. This more natural normalization convention differs from that adopted in previous works $[16,17]$.

Therefore, the amplitude for the bridge scattering $i \rightarrow k \rightarrow j$ is given by the product of amplitudes of the elementary two-AO scatterings through the bridge: $i \rightarrow k$ and $k \rightarrow j$. Since the Wiberg-type bond-order $M(i \rightarrow j \mid k)$ contribution due to this bridge scattering is proportional to the conditional probability of Eq. 18, its is also seen to be related to the product of the Wiberg bond-orders of the associated two-AO propagation stages [16]:

$$
\begin{aligned}
M(i \rightarrow j \mid k) & =M_{i, k} M_{k, j}, \quad M_{i, k}=\left(\gamma_{i, k}\right)^{2}, \quad M_{k, j}=\left(\gamma_{k, j}\right)^{2}, \\
P(i \rightarrow j \mid k) & =M(i \rightarrow j \mid k)\left(N_{(i \rightarrow j \mid k)}\right)^{2} / 4^{2} .
\end{aligned}
$$

This development for the probability propagation via a single-AO bridge can be straightforwardly extended to cover several AO in the bridge. Consider, e.g., the 
two-AO bridge in the sequential scattering $i \rightarrow k \rightarrow l \rightarrow j$ described by the conditional (bridge) probability

$$
\begin{aligned}
P(i \rightarrow j \mid k, l) & =\left|A_{(i \rightarrow j \mid k, l)}\right|^{2}=\left(d_{i, k} d_{k, l} d_{l, j}\right)^{2} /\left(d_{i, i} d_{k, k} d_{l, l}\right) \\
& =\left(d_{i, k} / \sqrt{d_{i, i}}\right)^{2}\left(d_{k, l} / \sqrt{d_{k, k}}\right)^{2}\left(d_{l, j} / \sqrt{d_{l, l}}\right)^{2} \\
& =\left|A_{i \rightarrow k}\right|^{2}\left|A_{k \rightarrow l}\right|^{2}\left|A_{l \rightarrow j}\right|^{2}=P(i \rightarrow k) P(k \rightarrow l) P(l \rightarrow j) .
\end{aligned}
$$

It is defined by the associated amplitude, the renormalized implicit derivative of $\chi_{j}^{b}$ on $\chi_{i}^{b}$ through $\chi_{k}^{b}$ and $\chi_{l}^{b}$ :

$$
A_{(i \rightarrow j \mid k, l)}=N_{(i \rightarrow j \mid k, l)}\left(\frac{\partial \chi_{k}^{b}}{\partial \chi_{i}^{b}}\right)\left(\frac{\partial \chi_{l}^{b}}{\partial \chi_{k}^{b}}\right)\left(\frac{\partial \chi_{j}^{b}}{\partial \chi_{l}^{b}}\right)=N_{(i \rightarrow j \mid k, l)} d_{i, k} d_{k, l} d_{l, j} .
$$

Here, the normalization constant,

$$
N_{(i \rightarrow j \mid k, l)}=1 /\left(d_{i, i} d_{k, k} d_{l, l}\right)^{1 / 2},
$$

satisfies the sum rule

$$
\sum_{j} P(i \rightarrow j \mid k, l)=P(i \rightarrow l \mid k)
$$

It again expresses the fact that the summation of the conditional probabilities over all possible final outputs $\{j\}$ in the sequential scattering events $i \rightarrow k \rightarrow l \rightarrow\{j\}$ must reproduce the conditional probability of the preceding step of the single AO-bridge: $i \rightarrow k \rightarrow l$. These probabilities are related to the corresponding Wiberg-type bond order

$$
\begin{aligned}
M(i \rightarrow j \mid k, l) & =M_{i, k} M_{k, l} M_{l, j}, \quad M_{l, j}=\left(\gamma_{l, j}\right)^{2}, \\
P(i \rightarrow j \mid k, l) & =M(i \rightarrow j \mid k, l)\left(N_{(i \rightarrow j \mid k, l)}\right)^{2} / 4^{3} .
\end{aligned}
$$

Of interest also are the parallel single-AO bridges [18], consisting of all basis functions $\chi$, which determine the AO scattering cascade between orbitals $\chi_{i}$ and $\chi_{j}$ in the bond system of the molecule, $i \rightarrow\{k\} \rightarrow j \equiv i \rightarrow \chi \rightarrow j$,

$$
P(i \rightarrow j \mid \chi)=\left|A_{(i \rightarrow j \mid \chi)}\right|^{2}
$$

defined by the amplitude

$$
A_{(i \rightarrow j \mid \chi)}=N_{(i \rightarrow j \mid \chi)} \sum_{k}\left(\frac{\partial \chi_{k}^{b}}{\partial \chi_{i}^{b}}\right)\left(\frac{\partial \chi_{j}^{b}}{\partial \chi_{k}^{b}}\right)=N_{(i \rightarrow j \mid \chi)} \sum_{k} d_{i, k} d_{k, j}=N_{(i \rightarrow j \mid \chi)} d_{i, j},
$$


where we have used the idempotency relation of Eq. 4. The normalization constant

$$
N_{(i \rightarrow j \mid \chi)}=1 / d_{i, i}
$$

then indeed assures that (see Eq. 14)

$$
P(i \rightarrow j \mid \chi) \equiv \sum_{k} P(i \rightarrow j \mid k)=P(i \rightarrow j) .
$$

This single-cascade development can be easily extended to probe the multiplecascade bridges [18]. Consider for example the probability scattering through the double-cascade $i \rightarrow\{k\} \rightarrow\{l\} \rightarrow j \equiv i \rightarrow \chi \rightarrow \chi^{\prime} \rightarrow j:$

$$
\begin{aligned}
P\left(i \rightarrow j \mid \chi, \chi^{\prime}\right) & =\left|A_{\left(i \rightarrow j \mid \chi, \chi^{\prime}\right)}\right|^{2} \\
A_{\left(i \rightarrow j \mid \chi, \chi^{\prime}\right)} & =N_{\left(i \rightarrow j \mid \chi, \chi^{\prime}\right)} \sum_{k} \sum_{l}\left(\frac{\partial \chi_{k}^{b}}{\partial \chi_{i}^{b}}\right)\left(\frac{\partial \chi_{l}^{b}}{\partial \chi_{k}^{b}}\right)\left(\frac{\partial \chi_{j}^{b}}{\partial \chi_{l}^{b}}\right) \\
& =N_{\left(i \rightarrow j \mid \chi, \chi^{\prime}\right)} \sum_{k} \sum_{l} d_{i, k} d_{k, l} d_{l, j}=N_{\left(i \rightarrow j \mid \chi, \chi^{\prime}\right)} d_{i, j},
\end{aligned}
$$

where again the normalization of Eqs. 14 and 30 gives:

$$
P\left(i \rightarrow j \mid \chi, \chi^{\prime}\right) \equiv \sum_{k} \sum_{l} P(i \rightarrow j \mid k, l)=P(i \rightarrow j)
$$

Therefore, the multiple cascades, with each step involving all basis functions, indeed conserve the stationary direct probability scattering of Eq. 14 [18].

\section{Conclusion}

The familiar direct bonding mechanism is associated with an accumulation of the electronic charge between bonded atoms. Being conditioned by the overlap between the interacting orbitals it is possible only at relatively short distances between AIM. The indirect bonding does not require a presence of such a bond-charge. It depends on the existence of the real bridge of chemically interacting orbitals contributed by bonded atoms between the interacting AO. As such it can be effected also at large separations between atoms, thus having profound implications for biological, supra-molecular and solid-state systems.

The bottom-line of this new mechanism is that chemical bonding between two AO can be realized despite the vanishing $\mathrm{CBO}$ matrix element coupling directly these basis functions in the molecule, provided that they both exhibit non-vanishing density matrix elements with the bridge basis functions. In other words, the two AO may exhibit the indirect chemical bonding when they strongly couple to other directly bonded basis functions. 
As we have shown in this work, such indirect interactions originate from the implicit dependencies between $\mathrm{AO}$ in the molecular bond system, due to their chemical coupling to other basis functions in the molecular system as a whole. In OCT these indirect bond components are generated via the through-bridge communications between AO. They generate the IT-covalency (communication noise) and IT-ionicity (information flow) components of the associated implicit bond-interactions in molecules, which complement the familiar explicit bond contributions. The latter can be probed by alternative information densities [5,6,21-24], localized via Electron Localization Function (ELF) [25-27] or using the Contra-Gradience (CG) density [6,28-31], and their multiplicities can be adequately quantified using the generalized quadratic indices of MO theory [32-41].

Open Access This article is distributed under the terms of the Creative Commons Attribution Noncommercial License which permits any noncommercial use, distribution, and reproduction in any medium, provided the original author(s) and source are credited.

\section{References}

1. C.E. Shannon, Bell Syst. Tech. J. 27(379), 623 (1948)

2. C.E. Shannon, W. Weaver, The Mathematical Theory of Communication (University of Illinois, Urbana, 1949)

3. N. Abramson, Information Theory and Coding (McGraw-Hill, New York, 1963)

4. P.E. Pfeifer, Concepts of Probability Theory, 2nd edn. (Dover, New York, 1978)

5. R.F. Nalewajski, Information Theory of Molecular Systems (Elsevier, Amsterdam, 2006)

6. R.F. Nalewajski, Information Origins of the Chemical Bond (Nova Science Publishers, New York, 2010)

7. R.F. Nalewajski, D. Szczepanik, J. Mrozek, Adv. Quant. Chem., in press

8. R.F. Nalewajski, J. Math. Chem. 47, 709 (2010)

9. R.F. Nalewajski, J. Phys. Chem. A 104, 11940 (2000)

10. R.F. Nalewajski, Int. J. Quantum Chem. 109, 425 (2009)

11. R.F. Nalewajski, Int. J. Quantum Chem. 109, 2495 (2009)

12. R.F. Nalewajski, Adv. Quantum Chem. 56, 217 (2009)

13. K.A. Wiberg, Tetrahedron 24, 1083 (1968)

14. R.F. Nalewajski, J. Math. Chem. 47, 692 (2010)

15. R.F. Nalewajski, J. Math. Chem. 49, 592 (2011)

16. R.F. Nalewajski, J. Math. Chem. 49, 371 (2011)

17. R.F. Nalewajski, J. Math. Chem. 49, 546 (2011)

18. R.F. Nalewajski, J. Math. Chem. 49, 806 (2011)

19. S. Shaik, D. Danovich, W. Wu, P.C. Hiberty, Nat. Chem. 1, 443 (2009)

20. P.A.M. Dirac, The Principles of Quantum Mechanics, 4th edn. (Clarendon, Oxford, 1958)

21. R.F. Nalewajski, E. Broniatowska, J. Phys. Chem. A. 107, 6270 (2003)

22. R.F. Nalewajski, E. Switka, A. Michalak, Int. J. Quantum. Chem. 87, 198 (2002)

23. R.F. Nalewajski, E. Świtka, Phys. Chem. Chem. Phys. 4, 4952 (2002)

24. R.F. Nalewajski, A.M. Köster, S. Escalante, J. Phys. Chem. A 109, 10038 (2005)

25. A.D. Becke, K.E. Edgecombe, J. Chem. Phys. 92, 5397 (1990)

26. B. Silvi, A. Savin, Nature 371, 683 (1994)

27. A. Savin, R. Nesper, S. Wengert, T.F. Fässler, Angew. Chem. Int. Ed. Engl. 36, 1808 (1997)

28. R.F. Nalewajski, Int. J. Quantum. Chem. 108, 2230 (2008)

29. R.F. Nalewajski, P. de Silva, J. Mrozek, in Kinetic Energy Functional, ed. by A. Wang, T. Wesołowski (World Scientific, Singapore, 2010), in press

30. R.F. Nalewajski, J. Math. Chem. 47, 667 (2010)

31. R.F. Nalewajski, P. de Silva, J. Mrozek, J. Mol. Struct.: THEOCHEM 954, 57 (2010)

32. M.S. Gopinathan, K. Jug, Theor. Chim. Acta (Berl.) 63(497), 511 (1983) 
33. K. Jug, M.S. Gopinathan, ed. by Z.B. Maksić Theoretical Models of Chemical Bonding, vol. II. (Springer, Heidelberg, 1990), p. 77

34. I. Mayer, Chem. Phys. Lett. 97, 270 (1983)

35. R.F. Nalewajski, A.M. Köster, K. Jug, Theoret. Chim. Acta (Berl.) 85, 463 (1993)

36. R.F. Nalewajski, J. Mrozek, Int. J. Quantum. Chem. 51, 187 (1994)

37. R.F. Nalewajski, S.J. Formosinho, A.J.C. Varandas, J. Mrozek, Int. J. Quantum. Chem. 52, 1153 (1994)

38. R.F. Nalewajski, J. Mrozek, G. Mazur, Can. J. Chem. 100, 1121 (1996)

39. R.F. Nalewajski, J. Mrozek, A. Michalak, Int. J. Quantum. Chem. 61, 589 (1997)

40. J. Mrozek, R.F. Nalewajski, A. Michalak, Polish J. Chem. 72, 1779 (1998)

41. R.F. Nalewajski, Chem. Phys. Lett. 386, 265 (2004) 